\title{
The demographics and outcomes of burn patients admitted to Worcester Hospital
}

\author{
N Blake, ${ }^{1}$ (D) Owart, ${ }^{1,2}$ iD RC Duvenage ${ }^{1,2}$ \\ ${ }^{1}$ Department of General Surgery, Worcester Provincial Hospital, South Africa \\ ${ }^{2}$ Ukwanda Centre for Rural Health, Stellenbosch University, South Africa
}

Corresponding author, email: drnikitablake@gmail.com

Background: Worcester Hospital is a regional healthcare facility in the Western Cape, South Africa, without a dedicated burns unit. Currently there is limited data available of burns patient management outside of academic institutions in South Africa. To describe the incidence and demographics, and to determine the outcomes of burn patients admitted to Worcester Hospital.

Methodology: A retrospective descriptive study of burn patients admitted to Worcester Hospital between 1 September 2016 and 31 August 2017.

Results: A total of 66 burn patients were included in this study which accounted for $1.6 \%$ of the total surgical admissions for this time period. The mean age of the patients was $39(\mathrm{SD} \pm 19)$ years with a male predominance $(59 \%)$. The mechanism of burn was mostly flame burns (71\%); 16 patients $(24 \%)$ were burned with hot fluids and 3 patients (5\%) sustained electrical burns. The median TBSA was 9\% (IQR: 5-28). Ten patients (15\%) required critical care unit admission. The burn patients' median length of stay was 6 days (IQR: 2-11 days) versus 2 days (IQR: 1-5 days) for non-burn general surgery patients. Fifty burn patients (76\%) required surgical intervention comprising of either debridement or skin grafting, or a combination of this. Forty-four patients $(67 \%)$ underwent skin grafting procedures and the median TBSA grafted was 5\% (IQR: 3.5-9.5). The median time from admission to first surgical procedure was 25 hours (IQR: 18.33-51.08). The in-hospital mortality rate was $23 \%$ and of the 15 mortalities, 9 patients $(60 \%)$ had TBSA of $30 \%$ or more and therefore classified as a major burn.

Conclusion: Burn injuries treated at Worcester Hospital are often severe and require significant resources. This study supplies critical information regarding the burden of burn related injuries managed at a regional level.

Keywords: burns, regional hospital, demographics, length of stay, mortality, low- to middle-income country (LMIC)

\section{Introduction}

Burn injuries cause mortality and severe morbidity and are more common in developing countries., ${ }^{1,2}$ Poor socioeconomic circumstances and housing environments contribute to the high incidences of burns and burn severity in Southern Africa. ${ }^{3,4}$ Epileptics and children being especially vulnerable to burn related injuries. ${ }^{5,6}$ Burn injuries are a massive burden on already an overloaded healthcare system in South Africa. ${ }^{7}$

Conservative management and even traditional alternatives for burn care have long been explored, but inevitably lead to delayed definitive management and longer hospital stay. ${ }^{8}$ An aggressive approach and early surgical management of burns have a more cost effective outcome than conservative options, even in settings with limited resources. ${ }^{7}$ Unfortunately, the costs involved in these treatments for individuals remain a concern and often an ethical dilemma when resources are slim for entire populations. ${ }^{9}$ Despite the obvious constraints faced by hospitals in South Africa, our burn resuscitation protocols are similar to international standards, ${ }^{10}$ yet morbidity and mortality remain higher compared to high-income countries. This could reflect a failure of implementation or failure of compliance to these protocols. Commonly used modified burn scores have been adjusted accordingly to predict mortality outcome in our setting. ${ }^{11}$ Delays to theatre, limited access to suitable dressings and complications such as pseudomonas infections often prolong hospital stay which in turn puts further strain on the healthcare system. ${ }^{12}$

In low- to middle-income countries (LMIC), the demand for ICU and tertiary care of burn patients exceeds the availability, as is the case in South Africa's public health service. ${ }^{13}$ Patients who should be managed in specialised burn units often have to wait at regional or district level hospitals until beds become available in the superior unit. This compromises the care needed and neglects various other support services deemed crucial. There is a movement for the reorganisation of burn services, specifically in the Western Cape, along with a strong multidisciplinary approach. ${ }^{14}$

The Western Cape provincial guidelines for the management of burn patients recommend tertiary care in a burns unit for all burns of partial-thickness more than $25 \%$ of total body surface area (TBSA) or full-thickness more than $15 \%$ TBSA, and for inhalation burns requiring ventilation for more than 48 hours. These guidelines also 
advise tertiary care for any burn in patients older than 60 years of age or patients with existing comorbidity. Serious mechanism of injury burns (e.g. electrical burns) or burns with severe associated injuries (e.g. polytrauma) should also be managed by a tertiary centre. ${ }^{15}$

There is limited data available of burn patient demographics and outcomes in the public health sector, especially outside of academic institutions in South Africa. This study aims to bring more clarity to challenges and outcomes of burn patients primarily managed at a regional hospital in the Western Cape.

\section{Methods}

A retrospective review of prospectively collected data was conducted of burn patients admitted at Worcester Hospital (WPH) over a one-year period. Worcester Hospital is a regional or level 2 healthcare facility with a 29-bed surgical ward and a 5-bed high care unit (HCU), and services a rural population of approximately 640000 in the Cape Winelands East and Overberg district of the Western Cape, South Africa. ${ }^{16}$ Burn patients requiring in-patient care are primarily managed by the general surgery team and no plastic or reconstructive services are available at this hospital.

The data of all burn patients admitted to Worcester Hospital surgical ward and HCU for the period of 1 September 2016 to 31 August 2017 was extracted from the Worcester Hospital Department of Surgery electronic patient database upon admission and discharge. All burns related diagnosis with or without a concurrent diagnosis were included in the study. The data collected comprised of demographic information, burn related characteristics, details of treatment, length of stay (LOS), complications and outcomes. Readmissions data was also included. Referring hospitals were numbered 1 to 9 and included Worcester Hospital emergency care unit. Time from admission to Worcester Hospital admission to first surgical procedure was used as a measure of access to definitive burn care.

Means with standard deviations for normally distributed data and medians with interquartile ranges for non-normally distributed data were calculated in Excel. Hypothesis testing was performed on numeric data using the unpaired t-test and on categorical data using the chi-square test. If the assumptions for the chi-square test were not met, the Fisher's exact test was used instead. Stata version 15.1 (Statacorp, Texas, USA) was used for statistical analysis.

\section{Results}

A total of 66 burn patients were admitted from 1 September 2016 to 31 August 2017. This accounted for $1.6 \%$ of all surgical admissions. Sixty-one patients $(92 \%)$ were 18 years or older, 2 patients $(3 \%)$ were between 13 and 17 years and only 3 patients $(5 \%)$ were 12 years old or younger. The predominant mechanism of burn was flame burns, occurring in $71 \%$ of admissions (47 patients). The median TBSA was 9\% (IQR: 5-28). While many burn patients present with mixed burns, we used the most severe burn present to classify the degree of burn sustained. The majority of patients admitted (83\%) had full-thickness burns as their most severe degree present. All superficial burns were treated as out-patients. Most patients were referred from Worcester Hospital emergency care unit (59\%) and Ceres Hospital contributed the second highest number of patients $(17 \%)$.
Delay at referring centre was defined as referral done more than 24 hours after presentation of the burn patient to their facility. The majority of patients $(83 \%)$ were referred timeously, but $11(17 \%)$ were delayed by more than 24 hours before being referred to Worcester Hospital general surgery department. Intravenous fluid resuscitation according to the Parkland formula was appropriately administered at the referring centre during the initial management of $26(70 \%)$ of the 37 patients with TBSA burns more than $10 \%$. Only 4 patients $(6 \%)$ admitted suffered from epilepsy. Thirty-five per cent of burn patients had significant comorbidities, these included mostly hypertension, asthma and HIV.

Ten $(15 \%)$ burn patients were admitted to HCU and all required ventilation. Three patients did not qualify for critical care unit admission due to the severity of their burns. These 3 patients were discussed with the tertiary burn unit and managed palliatively. The median number of days in Worcester Hospital HCU was 2 (IQR: 2-5 days). Regarding the median total days in hospital, burn patients (6 days) stayed significantly longer than non-burn general surgery patients (2 days) at Worcester Hospital. Readmissions had a long mean LOS of 11 days.

Fifty burn patients $(76 \%)$ required surgical intervention comprising of either debridement or skin grafting, or a combination of this. Debridement is used as a broad term and refers to scrubbing of all burns regardless of depth as well as tangential excision of full-thickness burns, usually with a humby knife. Twenty-three $(46 \%)$ of these 50 patients required only one surgical intervention and 54\% required two or more trips to theatre. Forty-four patients (67\%) underwent skin grafting procedures and the median TBSA grafted was 5\% (IQR: $3.5-9.5$ ). The mean number of units of red cell concentrate transfused was 6 units, \pm 2 per person for the 12 patients $(18 \%)$ requiring blood products.

A significant proportion of patients $(18 \%)$ required readmission. Fifteen patients $(23 \%)$ died and of the 15 mortalities, 9 patients $(60 \%)$ had TBSA of $30 \%$ or more and therefore classified as a major burn. The relationship between the mortality and the abbreviated burn severity index (ABSI) score is demonstrated in Table 2.

\begin{tabular}{lccc}
\multicolumn{4}{c}{ Table 2: Comparison mortality and ABSI score ${ }^{17}$} \\
\hline & ABSI 2-5 & ABSI 6-9 & ABSI $\geq \mathbf{1 0}$ \\
\hline Survived & 31 & 18 & 2 \\
Mortality & $0(0 \%)$ & $9(33 \%)$ & $6(75 \%)$ \\
Total & 31 & 27 & 8 \\
\hline
\end{tabular}

\section{Discussion}

This study provides a multitude of demographic information regarding burn patients managed at a regional level. Even though burn injuries are the minority of surgical admissions, the spectrum of burns managed at Worcester Hospital is vast, predominantly from thermal injuries in the adult population. Many patients who qualify for tertiary care are managed at regional level with varying outcomes. Major burns of more than 30\% TBSA have a high mortality in our setting. Smaller burns are treated early and efficiently, but all burn injuries put significant strain on resources. The shortage of ICU beds and specialised burns units forces smaller hospitals to manage patients that are beyond their scope of treatment. ${ }^{18}$

The contribution of burns to the total surgical population at Worcester Hospital is small $(1,6 \%)$, but requires intensive 
resources. Length of hospital stay, dressings, theatre time and blood transfusions all contribute significantly to the cost of burn care within a regional hospital with smaller budget allocations than tertiary institutions. ${ }^{7}$ While the study population included all burn patients, adult burn admissions were more representative of the spectrum and burden of disease. The predominance of adult male patients as well as flame burns as aetiology is in line with other studies. ${ }^{4,19}$ Interestingly only $6 \%$ of the patients in our study were epileptics who sustained their burns during a seizure, compared to $20 \%{ }^{6}$ and $50 \%{ }^{13}$ in similar studies in Kwa-Zulu Natal, possibly indicating better control of epilepsy in the Western Cape or simply a lower incidence thereof. ${ }^{13}$

The low rate of paediatric burn admissions at our centre is not a reflection of low incidence. Paediatric burn patients are often referred and accepted directly to the tertiary paediatric burns unit from referring centres thus bypassing the general surgery department at Worcester Hospital. Paediatric burns do not occur less frequently than adult burns, ${ }^{5,19}$ but seem to be accepted to tertiary care earlier in our setting.

In keeping with most burn units in LMICs, our referral tertiary adult burn unit has a limited number of beds, especially ICU beds, as well as inadequate theatre time for its patient load..$^{2,8,18}$ Many patients who meet local criteria for referral are managed at regional level. ${ }^{14}$ At Worcester Hospital we realised through personal experience we would often get adult burn patients to theatre for debridement and skin grafting before they were accepted to the tertiary burn unit. For this reason, we regularly manage burn patients fit for referral to a tertiary unit, but not for ICU, without contacting the tertiary unit. ICU patients are always discussed.

The TBSA burns managed at Worcester hospital ranged from $1-86 \%$, the median TBSA of $9 \%$ is a significant burn surface area, but less than the mean values available from comparative studies from another regional hospital in South Africa. The mean TBSA at this regional hospital in KwaZulu-Natal from 2006 to 2008 was $23 \%$ in adults and $7.5 \%$ in children, and another audit in the same hospital from 2012 to 2013 noted a mean TBSA of $13 \%$ for adults and $9 \%$ for children. ${ }^{13,18}$

Although our TBSA values are smaller, more of our burn admissions required surgical intervention, $76 \%$ compared with $45 \%$ in a similar study. ${ }^{13}$ We attempt the gold standard early debridement and grafting for all full-thickness burns that we get to theatre within 24 hours of the incident; if this is not possible, we debride only. ${ }^{20}$ Seventeen patients $(26 \%)$ were grafted during the first surgical procedure. In this study, the term "surgical procedure" is limited to procedures performed under general anaesthesia. Scrubbing in the emergency unit or ICU was not included, nor was any scrubbing under sedation. All patients were scrubbed prior to tangential excision while under general anaesthesia. Six patients had partial-thickness burns or burns that were undeclared extensive enough to require further evaluation under general anaesthesia. Tangential excision was either not required or extent of the burn was uncertain. We did include this in our surgical group as one of our primary goals was to evaluate time to theatre for burn patients. Forty-four patients did receive tangential excision and skin grafting.

Our results show that our time from admission to first surgical procedure is a median of 25 hours. We have determined that this is a measure of our quality of burn care and find this value to be favourable for a regional hospital.
We achieve this due to a 24-hour emergency list that is well administrated according to a triage system. Our theatre time is also not as severely affected by critical polytrauma patients as in bigger centres. This assists us in providing an acceptable service despite having no dedicated burn theatre slates. Allorto et al. found that the mean time from burn to skin graft at their regional hospital was 51 days, but does not specify the time from admission to surgery. ${ }^{13}$ The number of patients who did qualify for tertiary care but were successfully managed at our centre relieve the pressure from the overloaded tertiary unit, but ensue significant cost implications for the regional hospital. ${ }^{7}$ Our biggest clinical challenges are inhalation and severe burns and the HCU care involved with these patients, but logistically the cost and availability of silver dressings, as well as bed occupancy of burn patients, prove to be the biggest frustration.

The median LOS for a burn patient was 6 days compared to 2 days for a non-burn surgery patient. Therefore, a small surgical population group has significant impact on the functioning of a general surgery department. Other studies had mean LOS in hospital for burn patients that varied between 15 and 48 days. ${ }^{13,21}$ Twelve patients (18\%) required readmission for loss of skin graft or burns that failed to heal timeously with conservative management. One readmission was planned at the patient's request to delay management for personal issues. Of the 12 readmissions, 9 patients $(75 \%)$ had a longer LOS than the duration of the initial admission. The high readmission rate could also indicate that patients are being discharged too early. This is likely due to pressure to open up beds for other emergencies requiring admission.

The median LOS of the $10 \mathrm{HCU}$ patients was 2 days, after which patients either demised or were transferred to the tertiary unit; only 1 patient was discharged to the ward and then home 18 days later. This follows the provincial guidelines that inhalation burns requiring less than 48 hours ventilation can be managed at regional level, ${ }^{15}$ but simultaneously questions this practice if only 1 patient had an uncomplicated course of management.

According to a retrospective study at our tertiary adult referral burn unit, the mortality rate for the period 2003 to 2008 was $25 \%,{ }^{3}$ but we expect a regional hospital to have a lower rate due to treating less severe burns. A regional hospital in KwaZulu-Natal produced a mortality rate of $13 \%$ over a period of 18 months from 2012 to $2013 .{ }^{18}$ Thus, the mortality rate of $23 \%$ at Worcester Hospital is worrisome even when 3 of these 15 patients were for palliation only. Most of the mortalities were either waiting for a tertiary unit bed or too unstable to be transferred by the time they were accepted. All the deaths were adults, and of these deaths $60 \%$ had TBSA burns of more than $30 \%$, thus a major burn by definition.

The ABSI score was adopted due to its use in the tertiary unit. It is not used due to its accuracy, especially in the third world setting, ${ }^{11}$ but rather as a guideline to determine severe burns requiring transfer or to support the decision for palliation rather than active management, as was the case with 3 patients who did not qualify for ICU. The median ABSI score was 6 (IQR: 5-8) and classifies as moderately to severe burn injury. ${ }^{17}$

There is little comparative literature about burn management in regional hospitals in South Africa, but they seem to face similar challenges. A study at a regional hospital in South Africa, showed restricted access to tertiary care and 
are also managing burns requiring more specialised care and resources than they can provide. ${ }^{13}$ This beckons the question whether tertiary units should be addressed, or resources and training should be invested into regional hospitals. Either way, there are too few resources allocated to adult burn management and a deficit in doctors with substantial burn training in a continent in which burns occur so commonly. ${ }^{5}$

Limitations of this study were that we do not follow a standardised approach of referring every patient that qualifies for tertiary unit care according to local criteria and can thus not audit this accurately. Patients often have mixed burn injuries and it is difficult to document the exact percentage of full- versus partial-thickness burns. We also do not know the outcome of patients transferred to the tertiary unit and so we do not know the true mortality rate of our severe burns. No patients were excluded due to incomplete records.

The information gained through this audit is the first step in addressing regional burn care in the Western Cape, South Africa. Limited comparative studies are available from regional hospitals in other provinces and countries. Future prospective studies with standardised approaches for documenting referral criteria and outcomes are recommended.

\section{Conclusion}

Burn injuries remain a significant burden on healthcare in developing countries. The vast majority of burn research is published by tertiary units where most of the research is done, but it is likely that this grossly underestimates the burden of disease. Burn injuries treated at Worcester Hospital are often severe and qualify for tertiary care according to local criteria. Although the contribution of burns to the total surgical population is small, the severity of the injuries being treated at regional level requires intensive resources and generally has a longer LOS in hospital, thus increased cost. This is likely to be the case in many other regional hospitals in South Africa. This study supplies critical information regarding the burden of burn injuries managed at a regional level and should be considered for planning of appropriate resource allocation and management protocols.

\section{Conflict of interest}

The authors declare no conflict of interest.

\section{Funding source}

This research did not receive any specific grant from funding agencies in the public, commercial, or not-for-profit sectors.

\section{Ethical approval}

University of Stellenbosch Human Research Ethics Committee (\#N16/10/138).

\section{ORCID}

N Blake (iD) https://orcid.org/0000-0001-9333-2829
O Swart (iD https://orcid.org/0000-0003-0595-6688

\section{REFERENCES}

1. Zayakova Y, Vajarov I, Stanev A, Nenkova N, Hristov H. Epidemiological analysis of burn patients in East Bulgaria. Burns. 2014;40:683-8. https://doi.org/10.1016/j. burns.2013.08.016.

2. Tyson AF, Boschini LP, Kiser MM, et al. Survival after burn in a sub-Saharan burn unit: Challenges and opportunities.
Burns. 2013;39:1619-25. https://doi.org/10.1016/j. burns.2013.04.013.

3. Maritz D, Wallis L, Van der Merwe E, Nel D. The aetiology of adult burns in the Western Cape, South Africa. Burns. 2012;38:120-7. https://doi.org/10.1016/j.burns.2010.12.007.

4. Park JO, Shin SD, Kim J, Song KJ, Peck MD. Association between socioeconomic status and burn injury severity. Burns. 2009;35:482-90. https://doi.org/10.1016/j.burns.2008.10.007.

5. Cox SG, Martinez R, Glick A, Numanoglu A, Rode $\mathrm{H}$. A review of community management of paediatric burns. Burns. 2015;41:1805-10. https://doi.org/10.1016/j. burns.2015.05.024.

6. Faurie MP, Allorto NL, Aldous C, Clarke DL. A closer look at burn injuries and epilepsy in a developing world burn service. South African J Surg. 2015;53:3-5.

7. Allorto NL, Clarke DL, Thomson SR. A cost model case comparison of current versus modern management of burns at a regional hospital in South Africa. Burns. 2011;37:1033-7. https://doi.org/10.1016/j.burns.2011.04.004.

8. Albertyn R, Berg A, Numanoglu A, Rode H. Traditional burn care in sub-Saharan Africa: A long history with wide acceptance. Burns. 2015;41:203-11. https://doi.org/10.1016/j. burns.2014.06.005.

9. Rode H, Rogers A, Adams S, et al. The dilemma of treating major burns in South Africa. South African Med J. 2013;103:608-9. https://doi.org/10.7196/SAMJ.7361.

10. Rode H, Rogers AD, Cox SG, et al. Burn resuscitation on the African continent. Burns. 2014;40:1283-91. https://doi. org/10.1016/j.burns.2014.01.004.

11. Smith MTD, Allorto NL, Clarke DL. Modified first world mortality scores can be used in a regional South African burn service with resource limitations. Burns. 2016;42:1340-4. https://doi.org/10.1016/j.burns.2016.03.024.

12. Coetzee E, Rode H, Kahn D. Pseudomonas aeruginosa burn wound infection in a dedicated paediatric burns unit. South African J Surg. 2013;51:50-3. doi:10.7196/SAJS.1134.

13. Allorto NL, Oosthuizen GV, Clarke DL, Muckart DJ. The spectrum and outcome of burns at a regional hospital in South Africa. Burns. 2009;35:1004-8. https://doi.org/10.1016/j. burns.2009.01.004.

14. Rode H, Rogers AD, Numanoglu A, et al. A review of primary and secondary burn services in the Western Cape, South Africa. South African Med J. 2015;105:853-7. https://doi. org/10.7196/SAMJnew.8187.

15. Western Cape Government, Health Circular. H62 of 2011.

16. Statistics South Africa. Census 2011 Statistical release. Pretoria: Statistics South Africa; 2012.

17. Tobiasen J, Hiebert JM, Edlich RF. The abbreviated burn severity index. Ann Emerg Med. 1982;11:260-2. https://doi. org/10.1016/S0196-0644(82)80096-6.

18. Allorto NL, Clarke DL. Merits and challenges in the development of a dedicated burn service at a regional hospital in South Africa. Burns. 2015;41:454-61. https://doi. org/10.1016/j.burns.2014.07.021.

19. Blom L, Klingberg A, Laflamme L, Wallis L, Hasselberg M. Gender differences in burns: A study from emergency centres in the Western Cape, South Africa. Burns. 2016;42:1600-8. https://doi.org/10.1016/j.burns.2016.05.003.

20. Ong YS, Samuel M, Song C. Meta-analysis of early excision of burns. Burns. 2006;32:145-50. https://doi.org/10.1016/j. burns.2005.09.005.

21. Bauling PC, Kruger JS, Van der Vyver JD, Van Rooyen M, Lombard JH. Early benefits of improved burn-care capability at Kalafong Hospital. South African Med J. 1992;81:310-2. 